\title{
Prevalência de fatores associados à severidade dos acidentes em entorno de escolas
}

\author{
Tânia Batistela Torres ${ }^{1}$, Ana Margarita Larrañaga Uriarte ${ }^{2}$, Cristhiane Paludo Demore ${ }^{3}$, \\ Christine Tessele Nodari ${ }^{4}$
}

\begin{abstract}
${ }^{1}$ Laboratório de Sistemas de Transportes - LASTRAN, Universidade Federal do Rio Grande do Sul, batistela.torres@ufrgs.br 2Laboratório de Sistemas de Transportes - LASTRAN, Universidade Federal do Rio Grande do Sul, analarra@producao.ufrgs.br ${ }^{3}$ Laboratório de Sistemas de Transportes - LASTRAN, Universidade Federal do Rio Grande do Sul, cristhiane.demore@ufrgs.br ${ }^{4}$ Laboratório de Sistemas de Transportes - LASTRAN, Universidade Federal do Rio Grande do Sul, piti@producao.ufrgs.br
\end{abstract}

\section{Recebido:}

20 de fevereiro de 2017

Aceito para publicação:

07 de setembro de 2017

Publicado:

29 de outubro de 2017

Editor de área:

Flavio Cunto

\section{Palavras-chaves:}

Estrutura urbana,

Escola,

Logit Ordenado,

Logit multinomial,

Segurança viária.

\section{Keywords:}

Built environment,

School,

Ordered logit,

Multinomial Logit,

Road safety.

DOI:10.14295/transportes.v25i3.1331

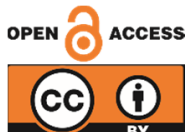

\begin{abstract}
RESUMO
Promover a segurança viária nos entornos escolares é uma estratégia que contribui para que sejam construídas cidades seguras, saudáveis e sustentáveis. Este estudo é dedicado a identificar o conjunto de características físicas urbanas capazes de reduzir a severidade dos acidentes nesses entornos. A severidade foi analisada a partir das características da estrutura urbana, socioeconômicas, da instituição escolar e dos acidentes registrados (entre 2012 e 2014) no entorno de 905 escolas de Porto Alegre. Foram estimados modelos de escolha discreta ordenados e não ordenados, permitindo a comparação entre as abordagens. Entretanto, não foi identificada superioridade de uma abordagem metodológica sobre a outra. O conjunto de estimativas indica que a severidade dos acidentes no entorno escolar é influenciada por características da escola, do acidente, socioeconômicas e pela estrutura urbana, com destaque para influência negativa do número de interseções com quatro vias.
\end{abstract}

\begin{abstract}
Fostering road safety nearby schools is a strategy that contributes to build safe, healthy and sustainable cities. The aim of this study is to identify the set of urban physical characteristics that are able to reduce the severity of accidents in these surroundings. Build environment, socioeconomic data, characteristics of the educational institution and accident data (from 2012 to 2014) nearby 905 schools in Porto Alegre were used to analyze severity. Discrete choice models of ordered and unordered structures were estimated and compared. However, it was not possible to identify the superiority of one methodological approach over the other. The models have shown that the severity of accidents near schools is influenced by school, accidents, socioeconomic characteristics and the build environment, in special the negative influence of the quantity of four-way intersections.
\end{abstract}

\section{INTRODUÇÃO}

Todos os anos milhares de vidas são interrompidas por acidentes de trânsito. Essas mortes chamam adicional atenção pela precocidade de suas vítimas. Atualmente, os acidentes já são a principal causa de morte entre jovens de 15 a 29 anos (WHO, 2015). Diariamente, mais de 500 crianças morrem e 20 mil ficam feridas em acidentes de trânsito em todo o mundo (Billingsley et al., 2015). Já no Brasil, a acidentalidade no trânsito foi a primeira causa externa de mortes entre crianças de 5 a 14 anos em 2014 (DATASUS, 2016).

A maior parte das mortes no trânsito ocorre em áreas urbanas, e afetam, principalmente, os usuários vulneráveis como pedestres e ciclistas (Welle et al., 2015). A estrutura das cidades e das relações que se 
estabelecem entre as pessoas que nela circulam afetam a acidentalidade. É possível que o espalhamento urbano (urban sprawl), propiciado pela expansão do uso do automóvel particular, seja uma das principais causas de morte no trânsito, principalmente para os pedestres (Ewing et al., 2003). Em oposição a esse modelo de desenvolvimento urbano que induz o aumento da motorização, a década de 90 foi marcada pelas estratégias de gestão da mobilidade (Ewing e Cervero, 2010). Entre as quais o New Urbanism e o Smart Growth são estratégias que têm a característica de estimular os modos ativos - a pé e bicicleta (VTPI, 2016).

Em 2016, a Organização das Nações Unidas (ONU) ressaltou que as cidades devem se tornar inclusivas, seguras, resilientes e sustentáveis (United Nations, 2016). A cidade sustentável, do ponto de vista de transportes, é a que consome menos recursos para garantir a mobilidade. A substituição do automóvel por modos ativos atua nesse sentido. Modos ativos são promovidos quando origens e destinos são aproximados - desenvolvendo menor dependência dos automóveis e menores velocidades - quando há mais incentivo a novos hábitos. No entanto, o receio de envolvimento em acidentes viários pode ser um empecilho à adoção desses modos. Estabelece-se a necessidade de melhor compreender quais elementos da estrutura urbana tornam as vias mais seguras e que podem estimular o uso de modos ativos. 0 planejamento urbano e de transportes orientado à segurança viária viabiliza a mobilidade sustentável.

A exposição ao risco a qual os usuários do meio urbano são submetidos junto à frequência com a qual os acidentes ocorrem resulta na severidade das lesões (Elvik e Vaa, 2004). Portanto, além da redução da frequência de acidentes, é necessário que seja minimizada a severidade daqueles acidentes que, porventura, ocorram. Nesse sentido, destaca-se a oportunidade de obter avanços na melhoria da segurança viária pela inclusão de características da estrutura urbana capazes de reduzir a exposição ao risco. A estrutura urbana como elemento influente na segurança viária é tipicamente analisada em cinco aspectos (5Ds): densidade, diversidade, desenho urbano, distância ao transporte coletivo e distância ao destino (Ewing e Cervero, 2010).

Do ponto de vista metodológico, a investigação da relação entre os 5Ds e a segurança viária pode ser feita pela estimação de modelos que os relacionem aos acidentes de diferentes severidades. Dessa forma, buscam-se identificar os conjuntos de características que descrevem cenários urbanos com diferentes níveis de acidentalidade. As principais abordagens na modelagem de severidade de acidentes são (Abay, 2013; Mannering e Bhat, 2014): (i) modelos que não consideram a natureza ordenada dos dados de severidade - tais como logit multinomial, logit hierárquico e logit misto de parâmetros aleatórios; e (ii) modelos que consideram a natureza ordenada dos dados de severidade - tais como probit e logit ordenados. Cada abordagem tem suas premissas e restrições, que causam implicações sobre as inferências dos modelos. A comparação empírica dos diferentes tipos de estruturas de modelos sugere que a superioridade de uma abordagem sobre outra pode depender muito dos dados utilizados, mas fornecem evidência empírica visando à generalização dos resultados para vários bancos de dados (Abay, 2013; Eluru, 2013; Ye e Lord, 2014; Mannering e Bhat, 2014).

Ciente do problema de segurança viária que atinge jovens e crianças em idade escolar e da oportunidade de promover estruturas urbanas seguras aos seus diferentes usuários, este trabalho tem dois objetivos principais: (i) identificar o conjunto de características da estrutura urbana que influenciam a severidade dos acidentes no entorno de escolas de educação básica de Porto Alegre; e (ii) comparar os resultados para os modelos de estruturas ordenadas e não ordenadas, contribuindo com evidência empírica para o estado da arte.

A maioria dos acidentes envolvendo crianças em idade escolar acontecem próximo às escolas (AbdelAty et al., 2007). Assim, é relevante e necessário compreender os fatores que afetam a segurança viária dessas áreas. Ainda que mais de $95 \%$ das mortes de crianças por lesões decorrentes de acidentes de trânsito ocorram em países de baixa e média renda (Peden et al., 2008), as iniciativas dedicadas a compreender as influências das características físicas do entorno escolar na segurança viária concentramse em países de alta renda. 
Este artigo está organizado em seis seções. A seção 2 apresenta experiências reportadas na literatura sobre elementos influentes na severidade das ocorrências; a seção 3 descreve o método adotado; a seção 4 apresenta os dados processados e a seção 5 descreve a estimação dos modelos e a discussão dos resultados obtidos. A seção 6 traz as conclusões do estudo.

\section{FATORES INFLUENTES NA SEVERIDADE DOS ACIDENTES NO ENTORNO DE ESCOLAS}

Os estudos da segurança viária em áreas escolares foram, principalmente, motivados pelas reduções nas taxas de crianças que exerciam mobilidade independente (MI) nas viagens escolares - iam a pé ou de bicicleta - entre os anos 60 e 2000. A mudança de hábito foi simultânea ao aumento das distâncias entre casa e escola e dos índices de viagens motorizadas (Orenstein et al., 2007; Andreou, 2010). A MI, situação na qual os jovens e as crianças se deslocam de forma autônoma (Hillman et al., 1990), está diretamente associada à adoção de modos ativos nessa faixa etária, dada a idade de habilitação para dirigir. 0 declínio do uso de modos ativos e o aumento da dependência do automóvel causou o aumento das distâncias médias percorridas pela frota de automóveis e foi acompanhado do aumento da acidentalidade (Ewing e Dumbaugh, 2009; Litman e Fitzroy, 2016).

Internacionalmente, os esforços no sentido de restaurar a MI e promover o transporte sustentável para a escola se deram, principalmente, pela implementação de programas do tipo Safe Route to School (SRTS). A redução das velocidades mostra-se necessária para a melhoria da segurança viária, dado o potencial de atenuação das lesões que podem proporcionar (Peden et al., 2008). É possível que a presença de crianças caminhando induza à ideia de que mais indivíduos estão expostos a maiores riscos, e que isso poderá resultar no aumento dos atropelamentos. No entanto, o aumento de viagens a pé não foi associado ao aumento desses acidentes quando as características da estrutura urbana e socioeconômicas foram incluídas (Jensen, 2008).

Os principais fatores de exposição ao risco se traduzem no volume de tráfego motorizado, nos conflitos de tráfego e na velocidade. A estrutura urbana, definida a partir do padrão de desenvolvimento urbano e do desenho viário é capaz de atuar sobre esses três fatores (Ewing e Dumbaugh, 2009) se constituindo, assim, em uma oportunidade concreta de melhorias na segurança viária desses espaços. As características da estrutura urbana têm sido expressas, inicialmente, por três dimensões: densidade, diversidade do uso do solo e desenho urbano (Cervero e Kockelman, 1997). Essas variáveis foram incluídas em 70\% dos estudos que se dedicaram a explicar a acidentalidade com crianças (Rothman, 2014). Às três dimensões iniciais foram adicionadas as relações do ambiente com o transporte coletivo e com a distância aos principais polos atratores de viagens, formando as cinco dimensões (5Ds): densidade, diversidade de uso do solo, desenho urbano, distância até o transporte público e acessibilidade ao destino (Cervero et al., 2009).

A qualidade da estrutura urbana no entorno da residência e da escola desempenha importante influência nas taxas de viagens de crianças para a escola por modo não motorizado (Curtis et al., 2015). 0 uso de modos ativos por indivíduos de idade escolar - tipicamente até 17 anos - representa o desenvolvimento da MI e desonera a dependência do uso do automóvel. Promover o transporte sustentável nos entornos escolares incentiva o uso dos modos ativos e reduz o uso do automóvel (Andreou, 2010). Com menor volume de veículos, esses entornos passam a ser mais seguros e atrativos aos modos ativos, dando origem a um ciclo virtuoso. Nesse ciclo, quanto maior a proporção de viagens por modos ativos, mais seguro fica o ambiente viário e quanto mais seguro é o ambiente viário, maior é a propensão ao uso de modos ativos.

A densidade do uso do solo mensura o interesse por uma área, medido pelas variáveis que quantificam população, habitações, empregos e área útil construída, ou pela combinação dessas variáveis (Ewing e Cervero, 2010). Em análise de entornos escolares a densidade populacional foi relacionada à maior severidade de acidentes (Clifton e Kreamer-Fults, 2007). Quando analisada em relação a atropelamentos, contribuiu para que os acidentes fossem menos severos, enquanto que, em relação a acidentes 
entre veículo e bicicleta, mostrou-se relacionada a acidentes mais severos (Zahabi et al., 2011). Já a densidade de empregos apresentou impacto positivo na segurança de ciclistas (Chen e Shen, 2016), atuando na redução da severidade.

Em relação ao uso do solo diversificado, os efeitos pró e contra a redução da severidade foram observados a partir de diferentes contextos de análise. Os acidentes envolvendo veículos e usuários não motorizados registrados em toda Montreal (Canadá) e nos entornos escolares de Maryland (EUA) foram influenciados a serem mais severos quando o uso do solo era mais diversificado (Clifton e Kreamer-Fults, 2007; Zahabi et al., 2011). Já na análise conduzida em Seattle (EUA), essa dimensão foi claramente relacionada à probabilidade de acidentes menos severos entre veículo e ciclistas (Chen e Shen, 2016). A dimensão desenho urbano é a forma como a malha viária está distribuída em uma área (Cervero e Kockelman, 1997) e também tem sido relacionada ao aumento e à redução da severidade para as diferentes mensurações e locais de análise. Assim como as dimensões destinos acessíveis e distância ao transporte coletivo.

O uso de variáveis de controle tem mostrado contribuir com a análise da relação entre as características físicas urbanas e a acidentalidade (Rifaat et al., 2011). Nesse sentido, a inclusão de características socioeconômicas tem se destacado (Rothman et al., 2015). Condições ambientais também têm demonstrado contribuir para a explicação da severidade de acidentes (Yasmin et al., 2014). Nesse sentido, acidentes ocorridos de noite estão associados a maior velocidade e à fadiga, já a luz natural do dia atua negativamente na severidade do acidente, uma vez que garante melhor visibilidade.

\section{MÉTODO}

Modelos de escolha discreta ordenados e não ordenados foram estimados para determinar o efeito dos fatores influentes na severidade dos acidentes no entorno de escolas. Ambas as abordagens são amplamente usadas na modelagem de severidade de acidentes. Modelos ordenados - tais como probit e logit ordenado - consideram a natureza ordenada dos dados de severidade, e têm sido cada vez mais utilizados na modelagem da severidade devido à natureza ordenada destes dados, geralmente expressos em diferentes categorias de severidade (Quddus et al., 2010; Ye e Lord, 2011; Ferreira e Couto, 2012; Abay, 2013; Eluru, 2013; Ye e Lord, 2014). Dessa forma, alternativas adjacentes compartilham alguma tendência comum, a qual depende da proximidade entre as alternativas; quanto mais próximas elas estão, maior a tendência compartilhada (Train, 2009). As alternativas adjacentes também podem compartilhar alguns efeitos não observados.

Os modelos não ordenados - tais como logit multinomial, logit hierárquico e logit misto de parâmetros aleatórios - não consideram a natureza ordenada dos dados de severidade. Eles são construídos na suposição de independência de alternativas irrelevantes (Independence of Irrelevant Alternatives - IIA). Essa propriedade implica que a razão entre as probabilidades de duas alternativas que fazem parte de um conjunto de escolha é constante e independente da existência de outras alternativas no conjunto, isto é, as alternativas são independentes e não compartilham efeitos não observados. Por isso, modelos de resposta não ordenada poderiam fornecer estimativas inconsistentes quando aplicados aos resultados de respostas ordenadas (Ben-Akiva e Lerman, 1985).

No entanto, existem algumas deficiências potencialmente associadas aos modelos ordenados quando aplicados à segurança viária (Abay, 2013; Mannering e Bhat, 2014). A mais problemática é a restrição de monotonicidade que as respostas ordenadas impõem sobre os dados, que orienta a forma como as variáveis independentes do modelo afetam as sucessivas categorias de probabilidade. Eluru et al. (2008) e Washington et al. (2003) apresentam discussões sobre esse tema na modelagem da severidade de acidentes. Além disso, os modelos de respostas ordenadas são mais sensíveis ao subregistro de acidentes (Ye e Lord, 2011).

Devido às restrições dos modelos ordenados, modelos de resposta não ordenadas foram alternativamente utilizados na análise da severidade de acidentes (Rifaat et al., 2011; Ye e Lord, 2011; Eluru, 2013; 
Ye e Lord, 2014). Embora esses modelos não capturem a natureza ordinal das respostas, eles permitem efeitos mais flexíveis das variáveis e não impõem restrições sobre os parâmetros e efeitos marginais das variáveis (Abay, 2013). A superioridade de uma abordagem metodológica sobre outra pode depender muito dos dados utilizados (Mannering e Bhat, 2014). Portanto, neste estudo, ambos os tipos de modelos foram usados: (i) logit ordenado (OL) e (ii) logit multinomial (MNL) - não ordenado.

0 modelo OL considera que a variável dependente é discreta e seus valores refletem uma ordenação. Os valores adotados para a severidade foram 1, 2 e 3 se: somente danos materiais (Sev1), vítima ferida (Sev2) ou vítima fatal (Sev3), respectivamente. A probabilidade generalizada do acidente estar em determinada categoria $m$ é dada pela Equação 1 .

$$
P(y=m)=L\left(\mu_{m}-\beta^{\prime} x\right)-L\left(\mu_{m-1}-\beta^{\prime} x\right)
$$

em que $L$ representa a distribuição logística acumulada (Equação 2) e os $\mu_{s}$ são os pontos de corte. Nesse caso, a variável dependente possui três categorias, portanto, existem dois pontos de corte. 0 vetor de parâmetros a ser estimado é $\beta^{\prime}$ e $x$ é o vetor de variáveis explicativas.

$$
L\left(\beta^{\prime} x\right)=\frac{e^{\beta^{\prime} x}}{1+e^{\beta^{\prime} x}}
$$

O MNL (McFadden, 1974) é motivado por uma base teórica ligeiramente diferente em comparação aos modelos OL. A função de propensão (análogo à utilidade) que determina a probabilidade de indivíduos estarem em uma categoria de severidade específica $m$ foi especificada como uma função linear das variáveis explicativas observadas $\left(x_{p}\right)$ e um componente estocástico não observado $\left(\varepsilon_{p m}\right)$ (Equação 3):

$$
S_{p m}^{*}=\beta_{p m} X_{p}+\varepsilon_{p m}
$$

Em que $\beta_{p m}$ é vetor de parâmetros das alternativas que pode variar entre os indivíduos $p$ e $\varepsilon_{p m}$ é o erro estocástico distribuído (iid Gumbel). Assim, a probabilidade de indivíduos estarem em uma categoria de severidade específica $m$ é dada pela Equação (4):

$$
P_{p m}=\frac{e^{S p m}}{\sum_{m=1}^{M} e^{S_{p m}}}
$$

A estimação dos modelos foi realizada pelo método de máxima verossimilhança, no programa computacional Biogeme (Bierlaire, 2003). Ambos os modelos foram estimados para duas variáveis dependentes de severidade de acidentes no entorno de escolas: Severidade de acidentes e Severidade veículo$U V$ (envolvendo ao menos um automóvel e um usuário vulnerável ativo - pedestre ou ciclista). A seleção das variáveis independentes incluídas no modelo foi baseada no processo de eliminação regressiva (backward elimination), usando um nível de significância de 0.10 , para reduzir a possibilidade de eliminar variáveis importantes. A presença de multicolinearidade entre as variáveis independentes foi analisada através do fator de inflação da variância (Variance Inflation Factor - VIF), que mede o grau de aumento do erro padrão das variáveis independentes em comparação a quando essas variáveis não estão fortemente correlacionadas. 0 valor limite sugerido para VIF é de 10, caso em que o erro padrão seria inflacionado em mais de 3 vezes (Hair et al., 2009).

Para permitir a comparação da importância das variáveis explicativas com diferentes escalas de medidas, foram calculados os efeitos marginais das variáveis dependentes em relação às variáveis explicativas. Valores positivos de $\beta^{\prime}$ significam que um aumento em $X_{i}$ gerará o aumento (respectivamente, redução) da probabilidade da severidade mais alta (respectivamente, menor). No entanto, não é óbvio o efeito que um valor positivo ou negativo de $\beta^{\prime}$ vai gerar nas probabilidades dos níveis intermediários (Mannering e Bhat, 2014). Nos OL o efeito marginal da probabilidade de um acidente ter uma severidade $m$ em relação a uma variável $x$ foi calculado como (Equação 5): 


$$
P(y=m) / \partial x=\left[L\left(\mu_{m}-\beta^{\prime} x\right)-L\left(\mu_{m-1}-\beta^{\prime} x\right)\right] \beta
$$

No caso de variáveis dummy o efeito foi calculado como a diferença da probabilidade resultante de um incremento da variável de 0 a 1 . Nos modelos MNL, o efeito marginal foi calculado de forma similar, como o incremento na probabilidade resultante de um incremento infinitesimal na variável $x$, sendo as probabilidades descritas na Equação 4.

\section{DADOS}

A severidade nos acidentes no entorno de escolas foi obtida a partir dos registros de acidentes ocorridos em Porto Alegre no período de 2012 a 2014 (EPTC, 2015). As variáveis dependentes são: Severidade de Acidentes totais e Severidade veículo-UV - que correspondem a acidentes envolvendo um veículo de passeio (automóvel) e ao menos um pedestre ou ciclista (usuários vulneráveis não motorizados). Ambas variáveis são de natureza qualitativa ordinal, compostas por três níveis: (i) Sev1: valor 1 para acidente com somente dano material; (ii) Sev2, valor 2 para acidentes com vítima ferida; (iii) Sev3: valor 3 para acidentes com vítima fatal.

Para identificar o padrão de ocorrência do entorno de uma instituição escolar foi adotada a área circular de raio (buffer ring) de 200 metros. Essa área foi definida por meio dos seguintes critérios: (i) distância média caminhada até a escola, e (ii) percentual máximo de acidentes que são potencialmente influenciados pela presença da escola - limitando a $60 \%$ das ocorrências. As principais áreas de observação adotadas na análise da segurança e da mobilidade ativa são delimitadas por um raio de 400 metros (Patricios, 2002). Entretanto, em Porto Alegre, 73\% das viagens a pé com motivo escola/educação são deslocamentos com distâncias menores que 400 metros (Larrañaga et al., 2014), indicando a adoção de buffer com raio menor. Na referida pesquisa as distâncias foram autorreportadas e os respondentes possuíam 14 anos ou mais, não considerando crianças menores que frequentam as escolas, e que poderiam caminhar distâncias inferiores às identificadas na pesquisa. Neste estudo, o uso do raio de 400 metros resultaria na análise de $91 \%$ dos acidentes da cidade, sendo necessário limitar este valor para avaliar as ocorrências provavelmente relacionadas às variáveis do entorno de cada unidade escolar. Adotando o limite máximo de $60 \%$ das ocorrências, e combinando com o critério anterior, foi adotado o buffer de 200 metros (56\% dos acidentes).

As variáveis independentes candidatas são as características presentes na área de um buffer de 200 metros do entorno das 905 escolas de Porto Alegre. As variáveis (Tabela 1) são classificadas em quatro tipos: (i) do acidente, (ii) da escola, (iii) da estrutura urbana e (iv) socioeconômicas. As variáveis referentes às dimensões da estrutura urbana (5Ds) e a relação entre escolas e acidentes, foram obtidas por processamento de dados em Sistema de Informação Geográfica (SIG). Dada a indisponibilidade de características individuais para os envolvidos nos acidentes, todas as variáveis socioeconômicas referemse à população residente na área de entorno escolar. 0 índice de diversidade corresponde a uma medida alternativa ao índice de entropia (Cervero e Kockelman, 1997). Diante da indisponibilidade das áreas atribuídas a cada tipo de uso do solo, o índice de diversidade (Equação 6) é calculado para a presença dos usos do solo.

$$
I_{d i v}=\frac{\left|\sum_{i} P i \times \ln (P i)\right|}{\ln (i)}
$$

Em que $i$ é uso do solo na área de buffer e $P_{i}$ é o percentual do uso $i$. Nesta estimativa foram incluídos os números de escolas, indústrias, comércios, serviços e domicílios para cada entorno de escola, resultando no referido índice, que varia entre 0 e 1. 
Tabela 1: Descrição das variáveis independentes candidatas

\begin{tabular}{|c|c|c|c|c|c|}
\hline \multirow{2}{*}{ Variável } & \multicolumn{2}{|r|}{ Totais } & \multicolumn{2}{|c|}{ Veículo-UV } & \multirow[b]{3}{*}{ Fonte } \\
\hline & Média & Desvio padrão & Média & Desvio padrão & \\
\hline Dependente & & & & & \\
\hline Severidade de Acidentes totais (N:32844) & 1.31 & 0.473 & - & - & EPTC \\
\hline Severidade Veículo-UV (N:1534) & - & - & 2.01 & 0.199 & $2012-2014$ \\
\hline \multicolumn{6}{|l|}{ Independente } \\
\hline \multicolumn{6}{|l|}{ Acidentalidade } \\
\hline Veículo pesado $(0 ; 1)$ & 0.20 & 0.40 & - & - & \\
\hline Dia útil $(0 ; 1)$ & 0.81 & 0.39 & 0.80 & 0.40 & \\
\hline Chuva $(0 ; 1)$ & 0.08 & 0.28 & 0.06 & 0.24 & \\
\hline $\operatorname{Dia}(0 ; 1): 7 h-18 h$ & 0.75 & 0.43 & 0.65 & 0.48 & \\
\hline Horário de Pico $(0 ; 1): 7 h-9 h$ ou $17 h-19 h$ & 0.23 & 0.42 & 0.26 & 0.44 & \\
\hline Interseção $(0 ; 1)$ & 0.34 & 0.47 & 0.14 & 0.35 & \\
\hline \multicolumn{6}{|l|}{ Escola } \\
\hline Escola particular $(0 ; 1)$ & 0.62 & 0.49 & 1.00 & 0.61 & \multirow{2}{*}{ SMED 2013} \\
\hline Matrículas ( $n$ o) & 440.83 & 550.01 & 2959.00 & 498.08 & \\
\hline \multicolumn{6}{|l|}{$\begin{array}{r}\text { Estrutura urbana - 5Ds } \\
\end{array}$} \\
\hline \multicolumn{6}{|l|}{ Densidade } \\
\hline Densidade populacional (hab/km²) & 9887.06 & 6634.41 & 10750.98 & 7324.93 & \multirow{2}{*}{ IBGE/2010 } \\
\hline Densidade de domicílios (dom $/ \mathrm{km}^{2}$ ) & 4135.48 & 3397.99 & 4514.17 & 3820.97 & \\
\hline \multicolumn{6}{|l|}{ Diversidade } \\
\hline Índice de diversidade (0-1) & 0.40 & 0.20 & 0.38 & 0.20 & SMED 2015 \\
\hline \multicolumn{6}{|l|}{ Desenho urbano } \\
\hline Largura de via $(m)$ & 9.83 & 0.76 & 9.85 & 0.78 & \multirow{10}{*}{ EPTC 2014} \\
\hline Arterial $(0 ; 1)$ & 0.53 & 0.50 & 0.47 & 0.50 & \\
\hline Declividade média (\%) & 0.03 & 0.02 & 0.03 & 0.02 & \\
\hline Comprimento médio de quadra $(\mathrm{km})$ & 0.09 & 0.03 & 0.09 & 0.04 & \\
\hline Parcela de extensão de vias arteriais (\%) & 0.19 & 0.17 & 0.18 & 0.17 & \\
\hline Extensão de corredor de ônibus (km) & 0.11 & 0.17 & 0.09 & 0.16 & \\
\hline Extensão de ciclovia $(\mathrm{km})$ & 0.01 & 0.07 & 0.02 & 0.08 & \\
\hline Interseções ( $n$ o) & 11.27 & 6.73 & 12.08 & 7.44 & \\
\hline Interseções com 4 vias ( $n$ o) & 3.56 & 2.77 & 3.70 & 2.98 & \\
\hline Semáforos ( $n \stackrel{\circ}{)}$ & 2.74 & 2.47 & 2.69 & 2.49 & \\
\hline \multicolumn{6}{|l|}{ Acessibilidade ao destino } \\
\hline Comércios e serviços ( $n$ o) & 213.44 & 429.41 & 284.57 & 618.05 & SMIC 2015 \\
\hline 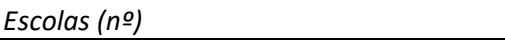 & 2.01 & 1.19 & 2.12 & 1.25 & SMED 2013 \\
\hline \multicolumn{6}{|l|}{ Disp. de transporte coletivo } \\
\hline Paradas de ônibus (no) & 5.14 & 5.89 & 6.14 & 8.05 & EPTC 2013 \\
\hline \multicolumn{6}{|l|}{ Socioeconômicas } \\
\hline Renda média (renda/dom) & 4301.46 & 2292.08 & 4111.45 & 2330.66 & \\
\hline Pessoas 4 -17 (\%) & 0.15 & 0.06 & 0.15 & 0.06 & IBGE/2010 \\
\hline Pessoas > 65 (\%) & 0.13 & 0.04 & 0.12 & 0.05 & \\
\hline
\end{tabular}

\section{RESULTADOS E DISCUSSÃO}

A Tabela apresenta os resultados dos modelos estimados para as variáveis dependentes Severidade de Acidentes totais e Severidade veículo-UV. Os coeficientes refletem os efeitos marginais das variáveis independentes sobre as chances de os acidentes apresentarem maior ou menor severidade. Coeficientes positivos indicam que uma variável aumenta a probabilidade da severidade do acidente, e os negativos indicam o oposto. 0 teste de VIF foi aplicado e foi identificada multicolinearidade entre as variáveis Densidade populacional e Densidade de domicílios, dentre as quais apenas Densidade populacional foi mantida, eliminando a presença de multicolinearidade ( $\mathrm{VIF}<10) .0$ ajuste geral dos modelos foi avaliado através do teste da razão de verossimilhança e o valor de Pseudo- $\mathrm{R}^{2}$, o qual varia entre 0 e 1 . Os modelos apresentaram ajuste satisfatório, já que valores próximos a 0,4 podem ser considerados muito bons ajustes (Ortúzar e Willumsen, 2011). Os valores obtidos são superiores aos reportados em trabalhos de severidade de acidentes (Abay, 2013). 
Tabela 2: Modelos e efeitos marginais (\%)

\begin{tabular}{|c|c|c|c|c|}
\hline \multirow[b]{3}{*}{ Variável } & \multicolumn{2}{|c|}{ Logit ordenado (OL) } & \multicolumn{2}{|c|}{ Logit multinomial (MNL) } \\
\hline & \multicolumn{2}{|c|}{ Sev(Ef1; Ef2; Ef3) } & \multicolumn{2}{|c|}{ (Ef1);Sev2( Ef2);Sev3(Ef3) } \\
\hline & Acidentes totais & Veículo-UV & Acidentes totais & Veículo-UV \\
\hline & & & $(14,07)-0,81(-13,88)$ & \\
\hline Chuva & & & $-0,87(-0,19)$ & \\
\hline Dia & $\begin{array}{c}-0.90(17,8 ;-17,30 ; \\
-0,50)\end{array}$ & $\begin{array}{c}-0,57(0,80 ; 0,57 \\
-1,37) \\
\end{array}$ & $\begin{array}{c}(17,96)-0,89(-17,53) \\
-1,26(-0,43)\end{array}$ & \\
\hline Dia útil & & & $\begin{array}{c}(4,56)-0,23(-4,35) \\
-0,54(-0,21)\end{array}$ & \\
\hline Horário de Pico & $\begin{array}{c}\mathbf{0 , 2 9} \\
(-5,41 ; 5,24 ; 0,17) \\
\end{array}$ & & $\begin{array}{c}(-5,66) 0,29(5,72) \\
(-0,06) \\
\end{array}$ & \\
\hline Interseção & $\begin{array}{c}-1,72 \\
(29,5 ;-28,9 ;-0,6)\end{array}$ & $\begin{array}{c}-1,51(3,54 ;-1,53 \\
-2,01)\end{array}$ & $\begin{array}{c}(31,35)-1,73(-30,96) \\
-1,79(-0,39)\end{array}$ & $\begin{array}{c}(13,46)-1.78(-13,13) \\
-2.72(-0,32)\end{array}$ \\
\hline Veículo pesado & $\begin{array}{c}-\mathbf{0 , 5 9} \\
(10,4 ;-10,1 ;-0,2)\end{array}$ & & $\begin{array}{c}(10,80)-0,61(-11,14) \\
0,39(0,34)\end{array}$ & \\
\hline Pré-escola & & $\begin{array}{l}\mathbf{0 , 9 2}(-1,61 ; \\
-0,35 ; 1,96)\end{array}$ & & \\
\hline Escola particular & $\begin{array}{c}-0,07 \\
(1,37 ;-1,33 ;-0,04)\end{array}$ & $\begin{array}{c}-0,86(2,04 ;-0,70 \\
-1,34)\end{array}$ & $\begin{array}{c}(1,27)-0,07 \\
(-1,28)(0,01) \\
\end{array}$ & $\begin{array}{c}(5,37)-0.73(-5,16) \\
-0.93(-0,2) \\
\end{array}$ \\
\hline $\begin{array}{l}\text { Índice de diversi- } \\
\text { dade }\end{array}$ & & & $\begin{array}{c}(4,91)-0,26 \\
(-4,96)(0,05)\end{array}$ & \\
\hline Arterial & $\begin{array}{c}\mathbf{0 , 0 5} \\
(-0,87 ; 0,84 ; 0,03)\end{array}$ & & $\begin{array}{c}(-0,07) \\
(-0,09) 0,48(0,16)\end{array}$ & \\
\hline $\begin{array}{l}\text { Comprimento mé- } \\
\text { dio de quadra }\end{array}$ & $\begin{array}{c}\mathbf{0 , 7 7} \\
(-14,2 ; 13,8 ; 0,4)\end{array}$ & & $\begin{array}{c}(20,18)-1,08 \\
(-20,39)(0,21)\end{array}$ & \\
\hline Interseções & $\begin{array}{c}\mathbf{0 , 0 2} \\
(-0,32 ; 0,31 ; 0,01)\end{array}$ & & $\begin{array}{c}(-0,66) 0,035(0,65) \\
\mathbf{0 , 0 3 8}(0,01)\end{array}$ & \\
\hline $\begin{array}{l}\text { Interseções com } 4 \\
\text { vias }\end{array}$ & $\begin{array}{c}-0,02 \\
(0,39 ;-0,38 ;-0,01)\end{array}$ & $\begin{array}{c}-0,08(0,12 ; 0,04 \\
-0,16)\end{array}$ & $\begin{array}{c}(-0,25) 0,015(0,29) \\
-0,096(-0,04)\end{array}$ & $\begin{array}{c}(1,5 \mathrm{E}-03)(1,8 \mathrm{E}-02) \\
-0.09 \\
(-1,98 \mathrm{E}-02) \\
\end{array}$ \\
\hline Largura de via & $\begin{array}{c}\mathbf{0 , 0 8} \\
(-1,48 ; 1,43 ; 0,05)\end{array}$ & & $\begin{array}{c}(-1,51) 0,08(1,52) \\
(-0,01)\end{array}$ & \\
\hline Semáforos & $\begin{array}{c}-0,04 \\
(0,80 ;-0,78 ;-0,02)\end{array}$ & & $\begin{array}{c}(-0,64) 0,04(0,67) \\
-0,07(-0,03)\end{array}$ & \\
\hline $\begin{array}{l}\text { Comércios e servi- } \\
\text { ços }\end{array}$ & & $\begin{array}{l}\text { 6.41E-4 (-9,9E-04; } \\
-4,7 \mathrm{E}-04 ; 1,5 \mathrm{E}-03) \\
\end{array}$ & & \\
\hline Escolas & & & $\begin{array}{c}(0,38)-\mathbf{0 , 0 2} \\
(-0,39)(0,004) \\
\end{array}$ & \\
\hline Paradas de ônibus & $\begin{array}{c}\mathbf{0 , 0 1} \\
(-0,19 ; 0,18 ; 0,01) \\
\end{array}$ & & $\begin{array}{c}(0,14)-0,008(-0,15) \\
0,03(0,01)\end{array}$ & \\
\hline Renda média & $\begin{array}{l}-5.2 E-05(9,6 E-04 ; \\
-9,3 E-04 ;-2,9 E-05)\end{array}$ & & $\begin{array}{l}(4,8 \mathrm{E}-04)-2.6 \mathrm{E}-05 \\
(-4,9 \mathrm{E}-04)(5 \mathrm{E}-06)\end{array}$ & \\
\hline Pessoas $4-17$ & & $\begin{array}{l}3,61(-5,51 ; \\
-2,90 ; 8,41)\end{array}$ & & \\
\hline N. de parâmetros & 13 & 7 & 17 & 3 \\
\hline Pseudo- $\mathrm{R}^{2}$ & 0.489 & 0.832 & 0.493 & 0.826 \\
\hline Log likelihood & -18433.484 & -269.623 & -18254.83 & -285.569 \\
\hline AIC & 36892.97 & 553.246 & 36543.66 & 577.14 \\
\hline
\end{tabular}

A comparação entre os modelos foi realizada pelo Akaike Information Criterion (AIC) (Akaike, 1992). Os valores obtidos para ambas as variáveis dependentes foram similares. Embora não se utilize teste estatístico para comparar AIC, a literatura recomenda que diferenças entre AIC maiores que 10 são suficientes para preferir um modelo sobre outro. Assim, para Severidade de Acidentes totais o modelo que apresentou melhor ajuste, dentre os estimados, foi o modelo MNL (menor valor de AIC). Entretanto, para Severidade Veículo-UV foi o modelo OL. Assim, por esse critério, não é possível afirmar superioridade de uma abordagem metodológica em relação à outra. Modelos de resposta ordenada e não ordenada apresentarem ajustes satisfatórios. 0 valor de AIC obtido na modelagem de Severidade Veículo-UV foi signifi- 
cativamente menor do que para Severidade de Acidentes totais, indicando um melhor ajuste dos dados. Os itens que seguem apresentam a interpretação dos parâmetros estimados e dos efeitos marginais para os modelos estimados.

\subsection{Severidade dos acidentes totais}

Na modelagem dos Acidentes totais seis variáveis relativas à estrutura urbana foram significativas para as duas técnicas de modelagem: Arterial, Comprimento médio de quadra, Interseções e Interseções com 4 vias, Largura de via, Semáforos, e Paradas de ônibus. Adicionalmente, no modelo MNL as variáveis Índice de diversidade e Escolas também foram significativas.

Os acidentes ocorridos em vias de alta hierarquia viária (Arterial) e mais largas (Largura de via) têm maior probabilidade de terem vítimas feridas e mortas. Essas variáveis atuam como proxy do volume de tráfego e das velocidades desempenhadas: maior volume e maior velocidade contribuem com a probabilidade de acidente mais severos. Esse resultado coincide com os reportados na literatura especificamente para entorno escolar (Clifton e Kreamer-Fults, 2007). Nesse sentido, os coeficientes obtidos para Comprimento médio de quadra e Interseções com 4 vias reforçam esse conceito. Quadras mais longas distanciam as interseções e propiciam maiores velocidades, aumentando a probabilidade de acidentes com vítimas. Esse resultado também coincide com resultados obtidos em outros trabalhos da literatura (Zahabi et al., 2011). Em relação à presença de semáforos, os resultados indicam que áreas de entrono escolar com maior quantidade de Semáforos têm menor probabilidade de acidentes com vítimas feridas e mortas, resultado semelhante ao reportado por Chen e Shen (2016) para Seattle (EUA). Esse resultado não surpreende, visto que a implantação de semáforos visa à diminuição de acidentes e separação de conflitos.

O coeficiente negativo para o Índice de diversidade indica que o entorno escolar com uso do solo mais diversificado contribui com a probabilidade de acidentes menos severos, com efeito marginal de maior magnitude na severidade de nível 1 - somente dano material. De forma semelhante, a presença de escolas no entorno, representada pela variável Escolas, mostrou aumentar a propensão a acidentes com somente dano material. Em relação à disponibilidade de transporte coletivo, os resultados mostram que o maior número de Paradas de ônibus contribui com o aumento da probabilidade de acidentes fatais, tanto no modelo OL, quanto no MNL, embora os modelos demonstrem influências distintas para os outros níveis de severidade. Na literatura, as variáveis relativas à diversidade do uso do solo (Índice de diversidade), com destinos acessíveis (Escolas) e curta distância ao transporte coletivo (Paradas de ônibus) são conhecidas pelo seu potencial de incentivar as viagens ativas, e consequentemente, contribuírem com a redução da motorização que aumenta a exposição ao risco. Por outro lado, o incentivo a viagens ativas pode expor usuários vulneráveis aos riscos da motorização já existente para alguns locais. Nesse sentido, para Porto Alegre, o desenvolvimento da mobilidade ativa a partir da influência da estrutura urbana direcionada a usuários ativos deve ser simultâneo a real redução do risco de exposição imposto pelos altos índices de motorização.

Analisando as características do acidente, os coeficientes obtidos para Horário de Pico e Interseção indicam que acidentes fora do horário de pico e ocorridos em interseção têm menor probabilidade de apresentarem feridos e mortos do que unicamente danos materiais. Esse resultado pode estar relacionado ao desenvolvimento de menores velocidades, provocando acidentes menos severos - relação claramente reportada na literatura (Abay, 2013; Noland e Quddus, 2005). Acidentes ocorridos fora do final de semana (Dia útil) tendem a ser menos severos. Essa relação já foi reportada na literatura para a severidade de acidentes com motociclistas em Singapura (Quddus et al., 2002). Ainda, é possível que essa relação decorra da densidade de tráfego e respectiva influência nas velocidades possibilitadas.

Acidentes envolvendo Veículos Pesados demonstraram contribuir com a redução da severidade dos acidentes, contribuindo com o aumento da probabilidade de ocorrer apenas dano material (Sev1), segundo os resultados obtidos pelo modelo ordenado. Enquanto que, através dos resultados do modelo 
multinomial, é possível observar coeficiente positivo para a Sev3, indicando que o envolvimento de veículo pesado no acidente contribui com aumento de $0,34 \%$ na probabilidade de morte. No entanto, o impacto na probabilidade de morte é muito menor que aquele observado para a probabilidade de ocorrer Sev1, com efeito de 10,80.

Quanto às condições ambientais do momento do acidente, a presença de Chuva e a condição de iluminação natural (Dia) contribuem com a probabilidade de os acidentes resultarem em somente dano material, com efeitos marginais positivos de 14,07 e 17,8 a 17,96, respectivamente, para a Sev1. Os dias chuvosos são afetados por condições de difícil visibilidade, de forma que é esperada a compensação do risco adicional imposto por essa condição pelo comportamento mais prudente do usuário (Elvik, 2006), resultando na redução da velocidade. Já os acidentes ocorridos de noite têm sido associados a maiores velocidades, fadiga e visibilidade reduzida, enquanto que acidentes ocorridos em condições naturais de iluminação têm sido relacionados a menores severidades (Rifaat et al., 2011).

Em relação às características da instituição escolar, a condição Escola Particular foi associada a menores severidades. Essa característica está relacionada à renda dos estudantes e, junto à variável Renda média - condição socioeconômica dos domicílios de entorno - indica que acidentes mais severos estão relacionados a menores rendas.

\subsection{Severidade dos acidentes envolvendo entre veículo e usuário vulnerável ativo}

Os resultados obtidos para Severidade veículo-UV indicam três variáveis significativas nos modelos OL e MNL: Interseção, Escola Particular e Interseções com 4 vias. Adicionalmente, a estimação pelo OL incluiu as variáveis Dia, Pré-escola, Comércios e serviços e Pessoas 4-17.

Dentre as variáveis da estrutura urbana, apenas Interseções com 4 vias e Comércios e serviços foram significativas. Os efeitos marginais para Interseções com 4 vias indicam que um aumento da quantidade desse tipo de interseção no entorno escolar reduz a probabilidade de acidentes com morte, mas tem impacto de coeficiente positivo na probabilidade de acidentes com feridos. Vias com interseções de 4 vias estimulam a realização de viagens a pé, aumentando a exposição de usuários vulneráveis, mas ao mesmo tempo podendo implicar em velocidades menores. Essa combinação pode explicar o fato de acidentes com feridos, mas com a diminuição de mortes, pois as velocidades desenvolvidas podem não ser tão significativas. Comparando as magnitudes dos efeitos marginais é possível observar que o impacto na diminuição de acidentes fatais é superior aos outros. Já o coeficiente positivo obtido para Comércios e serviços indica que essa característica contribui com o aumento da probabilidade de vítimas feridas nos acidentes de entorno de escolas. Essa relação converge com a análise dos entornos escolares realizada em Baltimore (EUA), na qual é reportado o aumento da severidade de atropelamentos para mais destinos acessíveis, mensurado pelo percentual de residências com acesso a Comércios e serviços (Clifton e Kreamer-Fults, 2007). Os efeitos marginais mostram que um aumento no número de comércios e serviços no entorno escolar reduz a probabilidade de acontecer acidentes só com danos materiais e feridos, enquanto que probabilidade de acidentes com morte aumenta (o impacto é positivo, de 0.00147). Nesse sentido, a presença de comércios e serviços estimula a realização de viagens a pé (Ewing e Cervero, 2010), possivelmente, aumentando a exposição ao risco de usuários vulneráveis.

As variáveis significativas relativas ao momento e local do acidente, Dia e Interseção, mostraram contribuição com a redução da severidade de Acidentes veículo-UV. Assim como em relação à severidade de Acidentes totais, acidentes ocorridos em condições de maior visibilidade apresentam menor severidade. Essa relação foi, também, reportada em relação a atropelamentos (Yasmin et al., 2014). Já a ocorrência do acidente em uma Interseção foi relacionada à maior probabilidade de ocorrer somente dano material, com efeitos marginal positivo para a Sev1. Esse resultado é análogo ao encontrado no modelo para o total de acidentes e, possivelmente, essa relação se deva às menores velocidades desempenhadas nesses locais.

Analisando as características da escola, Escola particular e Pré-escola foram variáveis significativas. 
Enquanto a condição particular, assim como para Acidentes totais, foi relacionada à redução da severidade, a presença da etapa escolar Pré-escola foi relacionada ao aumento da probabilidade, principalmente de o acidente resultar em vítima fatal (Sev3). Essa variável indica a presença de mais usuários vulneráveis em condições de desenvolvimento cognitivo e indica aumento da exposição ao risco. Nesse mesmo sentido, a parcela de residentes em idade escolar (Pessoas 4-17) no entorno das instituições analisadas mostrou o maior impacto na probabilidade de acidente com morte (Sev3).

\subsection{Comparação modelos ordenados e não ordenados}

O modelo MNL apresentou melhor ajuste para a severidade de Acidentes totais, enquanto que o OL apresentou melhor ajuste para a severidade de Acidentes veículo-UV. No modelo MNL puderam ser incluídas mais variáveis para a severidade de Acidentes totais, no entanto, para a modelagem de Acidentes-UV, o OL pode incluir mais variáveis significativas. Assim, não é possível afirmar superioridade de uma abordagem metodológica em relação à outra. Em relação aos efeitos marginais computados para os modelos OL e MNL, foram verificadas diferenças significativas (Tabela 2). Por exemplo, analisando a categoria de acidentes com vítimas fatais $\left(E f_{3}\right)$ nos modelos para Severidade de Acidentes totais, é possível observar que os efeitos marginais de Interseções com 4 vias e Semáforos computados no OL são muito inferiores aos calculados com o MNL. Essa subestimação está presente também em outras variáveis. Essas diferenças são muito importantes na implantação de políticas de segurança viária, subestimar efeitos implica subestimar o impacto de mudanças em determinadas características. A diferença entre os efeitos marginais se deve ao fato que nos modelos OL os efeitos são distribuídos entre as diferentes categorias de severidade. Já o MNL é capaz de acomodar os efeitos de forma mais flexível, variando os impactos da mesma variável entre as diferentes categorias de severidade.

\section{CONCLUSÕES}

Este trabalho buscou identificar o conjunto de características da estrutura urbana que influenciam a severidade dos acidentes no entorno de escolas de educação básica de Porto Alegre e comparar os resultados para os modelos de estruturas ordenadas e não ordenadas. Além das características relativas à estrutura urbana, esta análise se beneficiou da inclusão de variáveis que descrevem as condições de ocorrência do acidente, socioeconômicas e da instituição escolar. Denominadas variáveis de controle, essas variáveis contribuíram com a análise da severidade, embora não se possa atuar sobre elas pela aplicação de medidas de engenharia. Os resultados mostram que as características da estrutura urbanas, da instituição escolar, socioeconômicas do entorno escolar, ambientais e de local do acidente influenciam a severidade dos acidentes. Interseção, Interseções com 4 vias e Escola Particular foram variáveis significativas para a severidade de Acidentes totais e de Acidentes veículo-UV nos modelos OL e MNL. Os resultados permitiram verificar os principais resultados empíricos já reportados para estrutura urbana em relação à segurança viária, e indicam que o desenvolvimento da mobilidade ativa a partir da influência da estrutura urbana direcionada a esses usuários deve ser simultâneo a real redução do risco de exposição imposto pelos altos índices de motorização.

A comparação das estruturas de modelos testadas não permitiu afirmar superioridade de uma abordagem metodológica sobre a outra. Os resultados mostram que existem diferenças significativas nos efeitos marginais para as estruturas, as quais podem ter um grande impacto na implantação de políticas de segurança viária. Assim, há benefícios na estimação de ambas estruturas, evitando resultados incompletos e inferências equivocadas.

\section{REFERÊNCIAS BIBLIOGRÁFICAS}

Abay, K. A. (2013) Examining pedestrian-injury severity using alternative disaggregate models. Research in Transportation Economics, v. 43, n. 1, p. 123-136. DOI: 10.1016/j.retrec.2012.12.002

Abdel-Aty, M.; S. Srinivas e C. Lee (2007) Geo-spatial and log-linear analysis of pedestrian and bicyclist crashes involving school-aged children. Journal of Safety Research, v. 38, p. 571-579. DOI: 10.1016/j.jsr.2007.04.006 
Akaike H. (1992) Information Theory and an Extension of the Maximum Likelihood Principle. In: Kotz S. e N. L. Johnson (eds) Breakthroughs in Statistics. Springer Series in Statistics (Perspectives in Statistics). Springer, New York, NY. DOI: 10.1007/978-1-4612-0919-5_38

Andreou, M. (2010) Planning for Pedestrian Safety around Schools. 96 f. Trabalho de Diplomação. (Faculty of the Built Environment), University of New South Wales, Sydney, Austrália. 2010.

Ben-Akiva, M. e S. Lerman (1985) Discrete Choice Modeling: Theory and Applications to Travel Demand. MIT Press. Massachusetts. ISBN 0-262-02217-6

Bierlaire, M. (2003). Biogeme: A free package for the estimation of discrete choice models, Proceedings of the 3rd Swiss Transportation Research Conference, Ascona, Switzerland.

Billingsley, S.; A. Silverman; M. Adhikari; R. Clarke e M. Cutler (2015) Goals for change, partners for action goals for action. Fia Foundation, Annual Report 2015. Obtido de: <https://www.fiafoundation.org/media/203498/fiaf-annual-report-2015spreads.pdf>. Acesso em 9 de julho de 2017.

Cervero, R. e K. Kockelman (1997) Travel demand and the 3Ds : density, design, diversity. Transportation Research Part C: Emerging Technologies, v. 2, n. 3, p. 199-219. DOI: 10.1016/S1361-9209(97)00009-6

Cervero, R; O. L. Sarmiento; E. Jacoby; L. F. Gomez e A. Neiman (2009) Influences of Built Environments on Walking and Cycling: Lessons from Bogotá. International Journal of Sustainable Transportation, v. 3, n. 4, p. 203-226. DOI: 10.1080/15568310802178314

Chen, P. e Q. Shen (2016) Built environment effects on cyclist injury severity in automobile-involved bicycle crashes. Accident Analysis and Prevention, v. 86, p. 239-246. DOI: 10.1016/j.aap.2015.11.002

Clifton, K. J. e K. Kreamer-Fults (2007) An examination of the environmental attributes associated with pedestrian-vehicular crashes near public schools. Accident Analysis and Prevention, v. 39, n. 4, p. 708-715.

Curtis, C.; C. Babb e D. Olaru (2015) Built environment and children's travel to school. Transport Policy, v. 42, p. 21-33. DOI: 10.1016/j.tranpol.2015.04.003

DATASUS (2016) Obtido de: <http://tabnet.datasus.gov.br/cgi/deftohtm.exe?sim/cnv/> Acesso em 24/05/2016.

Eluru, N.; C. R. Bhat C. R. e D. A. Hensher (2008) A mixed generalized ordered response model for examining pedestrian and bicyclist injury severity level in traffic crashes. Accident Analysis and Prevention, v. 40, n. 3, p. 1033-1054. DOI:10.1016/j.aap.2007.11.010

Eluru, N. (2013) Evaluating alternate discrete choice frameworks for modeling ordinal discrete variables. Accident Analysis and Prevention. v. 55, p. 1-11. Washington, D.C., DOI: 10.1016/j.aap.2013.02.012

Elvik, R. e T. Vaa (2004) The handbook of road safety measures. Elsevier, Oxford, Inglaterra.

Elvik, R. (2006) Laws of accident causation. Accident Analysis and Prevention, v. 38, n. 4, p. 742-747. DOI: 10.1016/j.aap.2006.01.005

EPTC (2015) Conjunto de Dados. Datapoa. Empresa Pública de Transporte e Circulação. Disponível em: <http://datapoa.com.br/dataset?q=acidentes\&sort=score+desc\%2C+metadata_modified+desc $>$ Acesso em 30 abril 2015.

Ewing, R. e R. Cervero (2010) Travel and the built environment: a meta-analysis. Journal of the American Planning Association. v. 76, n. 3, p. 265-294, 2010. DOI: 10.1080/01944361003766766

Ewing, R. e E. Dumbaugh (2009) The Built Environment and Traffic Safety: A Review of Empirical Evidence. Journal of Planning Literature, v. 23, n. 4, p. 347-367. DOI: 10.1177/0885412209335553

Ewing, R.; R. A. Schieber e C. V. Zegeer (2003) Urban sprawl as a risk factor in motor vehicle occupant and pedestrian fatalities. American Journal of Public Health, v. 93, n. 9. p. 1541-1545.

Ferreira, S. e A. Couto (2012) Categorical Modeling to Evaluate Road Safety at the Planning Level. Journal of Transportation Safety \& Security, v. 4, n. 4, p. 308-322. D0I: 10.1080/19439962.2012.679385.

Hair, J. F.; W. C. Black; B. J. Babin; R. E. Anderson e R. L. Tatham (2009). Análise multivariada de dados. Bookman Editora.

Hillman, M.; J. Adams e J. Whitelegg (1990) One false move: A Study of Children's Independent Mobility. Policy Studies Institute. London, UK.

Jensen, S. U. (2008) How to obtain a healthy journey to school. Transportation Research Part A: Policy and Practice, v. 42, n. 3, p. 475-486. DOI: 10.1016/j.tra.2007.12.001

Larrañaga, A. M. L.; L. I. Rizzi; J. Arellana; O. Stramb e H. B. B. Cybis (2014) The Influence of built environment and travel attitudes on walking: a case study of Porto Alegre, Brazil. International Journal of Sustainable Transportation. v. 10 n. 4, p. 332 342. DOI: $10.1080 / 15568318.2014 .933986$

Litman, T. e S. Fitzroy (2016) Safe Travels: Evaluating Mobility Management Traffic Safety Impacts. Victoria: Victoria Transport Policy Institute.

Mannering, F. L. e C. R. Bhat (2014) Analytic methods in accident research analytic methods in accident research: methodological frontier and future directions. Analytic Methods in Accident Research. v. 1, p. 1-22. DOI: 10.1016/j.amar.2013.09.001

McFadden, D. (1974) The measurement of urban travel demand. Journal of Public Economics, v. 3, n. 4, p.303-328. DOI: 10.1016/0047-2727(74)90003-6

Noland, R. B. e M. A. Quddus (2005) Congestion and safety: A spatial analysis of London. Transportation Research Part A: Policy and Practice, v. 39, n. 2005, 737-754. DOI: 10.1016/j.tra.2005.02.022

Orenstein, M. R.; N. Gutierrez; T. M. Rice; J. F. Cooper e D. R. Ragland (2007) Safe Routes to School Safety and Mobility Analysis. California: California Departament of Transportation.

Ortúzar, J. de D. e L. G. Willumsen (2011) Modelling Transport. JohnWiley \& Sons, Ltd. 4th ed. 
Patricios, N. N. (2002) Urban design principles of the original neighbourhood concepts. Urban Morphology. v. 6, n. 1, p. $21-32$. ISSN: $1027-4278$

Peden, M.; K. Oyegbite; J. Ozanne-Smith; A. A. Hyder; C. Branche; A. F. Rahma; F. Rivara e K. Bartolomeos (2008) World report on child injury prevention. Genebra: World Health Organization.

Quddus, M. A.; R. B. Noland e C. C. Hoong (2002) An analysis of motorcycle injury and vehicle damage severity using ordered probit models. Journal of Safety Research, v. 33, n. 4, p. 445-462. DOI: 10.1016/S0022-4375(02)00051-8

Quddus, M. A.; C. Wang e S. G. Ison (2010) Road Traffic Congestion and Crash Severity: Econometric Analysis Using Ordered Response Models. Journal of Transportation Engineering, v. 136, n. 5, p. 424-435. DOI: 10.1061/(ASCE)TE.19435436.0000044

Rifaat, S. M., R. Tay e A. De Barros (2011) Effect of street pattern on the severity of crashes involving vulnerable road users. Accident Analysis and Prevention, v. 43, n. 1, p. 276-283. DOI:10.1016/j.aap.2010.08.024

Rothman, L. M. (2014) Child pedestrian-motor vehicle collisions and walking to school in the city of Toronto: The role of the built environment. $199 \mathrm{f}$. Tese (Ph.D), The Institute of Medical Science: University of Toronto. Toronto, Canada.

Rothman, L. M.; R. Buliung; T. To; C. Macarthur; A. Macpherson e A. Howard (2015) Associations between parents perception of traffic danger, the built environment and walking to school. Journal of Transport and Health. v. 2, n. 3, p. 327-335. DOI: 10.1016/j.jth.2015.05.004

Train, K. E. (2009). Discrete choice methods with simulation. Cambridge University Press.

United Nations (2016) Goal 11: Make cities inclusive, safe, resilient and sustainable. Sustainable Development Goals: 17 Goals to transform our world. Obtido de: <http://www.un.org/sustainabledevelopment/cities/>. Acesso em 11 de maio de 2016.

VTPI (2016) Online TDM Encyclopedia. Canadá, 2014. Victoria: Victoria Transport Policy Institute. Disponível em: <http://www.vtpi.org/tdm/index.php>. Acesso em 24 de agosto de 2016.

Welle, B.; W. Li; C. Adriazola; R. King; M. Obelheiro; C. Sarmiento e Q. Liu (2015) Cities safer by design: guidance and examples to promote traffic safety through urban and street design. World Resources Institute. ISBN: 978-1-56973-866-5. Washington, DC, Estados Unidos.

Washington, S. P.; M. G. Karlaftis e F. L. Mannering (2003) Statistical and Econometric Methods for Transportation Data Analysis. Chapman \& Hall/CR, Boca Raton, Florida.

WHO (2015) Relatório Global Sobre o Estado da Segurança Viária 2015. World Health Organization. Genebra, Suíça. Obtido de: <http://www.who.int/violence_injury_prevention/road_safety_status/2015/Summary_GSRRS2015_POR.pdf?ua=1>/. Acesso em 9 de julho de 2017.

Yasmin, S.; N. Eluru e S. V. Ukkusuri (2014) Alternative ordered response frameworks for examining pedestrian injury severity in New York City. Journal of Transportation Safety and Security. v. 6, n. 4, p. 275-300. DOI: 10.1080/19439962.2013.839590

Ye, F. e D. Lord (2011) Investigation of effects of underreporting crash data on three commonly used traffic crash severity models: multinomial logit, ordered probit, and mixed logit. Transportation Research Record: Journal of the Transportation Research Board, n. 2241, Transportation Research Board of the National Academies, Washington, D.C., 2011. p. 51-58. DOI: 10.3141/2241-06

Ye, F. e D. Lord (2014) Comparing three commonly used crash severity models on sample size requirements: Multinomial logit, ordered probit and mixed logit models. Analytic Methods in Accident Research, v. 1, p. 72-85, DOI: 10.1016/j.amar.2013.03.001

Zahabi, S. A. H.; J. Strauss, K. Manaugh e L. F., Miranda-Moreno, L. F. (2011) Estimating Potential Effect of Speed Limits, Built Environment, and Other Factors on Severity of Pedestrian and Cyclist Injuries in Crashes. Transportation Research Record: Journal of the Transportation Research Board, n. 2247, p. 81-90. DOI: 10.3141/2247-10. 